\title{
AN INVESTIGATION OF THE $1.4 \mathrm{BeV} / \mathrm{c}^{2}$ NUCLEON ISOBAR IN PROTON-PROTON INTERACTIONS*
}

\author{
T. H. Tan, M. L. Perl, F. Martin \\ Stanford Linear Accelerator Center \\ Stanford University, Stanford, California \\ W. Chinowsky, R. Kinsey, $\dagger$ S. Klein, P. Schmidt \\ Lawrence Radiation Laboratory \\ University of California, Berkeley, California \\ M. Mandelkern, J. Schultz \\ Physics Department \\ University of California, Irvine, California
}

\begin{abstract}
The production of $N^{*}(1400)$ isobar in the reaction $\mathrm{pp} \rightarrow \mathrm{p} \mathrm{N}^{*^{+}}(1400)$, where $N^{*}(1400) \rightarrow n \pi^{+}$and $\mathrm{p} \pi^{\circ}$, is investigated with the aid of one pion exchange model. The one-pion exchange mechanism does not secm to dominate the production process. The isospin of $N^{*}(1400)$ is found to be $I=1 / 2$, and the elasticity of the resonance is estimated to be .66 .
\end{abstract}

(to be submitted to Phys. Rev. Letters)

\footnotetext{
*Work supported in part by the U.S. Atomic Energy Commission.

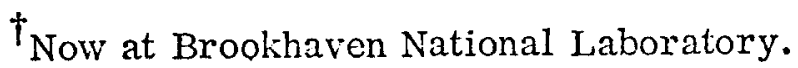


There has been considerable interest in the mass region near $1.4 \mathrm{BeV} / \mathrm{c}^{2}$ in the pion-nucleon system. A $\mathrm{P}_{11}$ resonance is required in all the $\pi-\mathrm{p}$ phase shift analyses of elastic scattering, ${ }^{1}$ yet in direct observation in production reactions, such a resonance has proved somewhat elusive. ${ }^{2}$ One of the strongest effects has been observed in the missing mass spectrum of the reaction $p+p \rightarrow p+$ missing mass, which at small four-momentum transfer exhibits a large enhancement near $1.4 \mathrm{BeV} / \mathrm{c}^{2} .{ }^{3}$ However, its identification with the $\mathrm{P}_{11}$ resonance has not been established. There has also been a suggestion that this enhancement may have a kinematic origin. ${ }^{4}$ To investigate the problem, we have studied the states $\mathrm{pn} \pi^{+}$and $\mathrm{pp} \pi^{\circ}$ in proton-proton collisions at $6.07 \mathrm{BeV} / \mathrm{c}$ in the LRL $72^{\prime \prime}$ hydrogen bubble chamber, in order to isolate for detailed examination two of the final states from all those summed over in the missing mass spectrometer experiments. This analysis was carried out with the aid of a one pion-exchange model.

Approximately 75,000 two-prong events were measured, about three-fourths with conventional film plane digitizing machines and the rest with the Lawrence Radiation Laboratory Flying Spot Digitizer. The events were fitted to the hypotheses

$$
\begin{aligned}
\mathrm{p}+\mathrm{p} & \longrightarrow \mathrm{p}+\mathrm{n}+\pi^{+} \\
& \longrightarrow \mathrm{p}+\mathrm{p}+\pi^{\mathrm{o}} \\
& \rightarrow \mathrm{p}+\mathrm{p}
\end{aligned}
$$

and those consistent with elastic scattering were eliminated from the sample. In addition, for reaction (1), it was required that the proton be traveling backward, with respect to the beam direction, in the total center-of-mass system. These protons were visually identified on the scanning table and, as a consequence, fitting ambiguities between (1) and (2) became negligible. After suitable 
correction for efficiencies and contamination, we found the cross sections for reactions (1) and (2) to be $6.7 \pm 0.5 \mathrm{mb}$ and $2.8 \pm 0.3 \mathrm{mb}$ respectively.

Since the data will be compared with a one-pion exchange model, we have eliminated the events in the region where the model is weakest, namely when both final state nucleons travel in the same direction with respect to the beam proton. There is strong evidence that a significant number of these events were misidentified, being in fact examples of multi-pion production with two unobserved. neutral particles. The removal of these events does not affect the $\pi$-nucleon mass spectra below $1.8 \mathrm{BeV} / \mathrm{c}^{2}$. The production of $\mathrm{N}^{*^{++}}$(1238) dominates reaction (1) and we have therefore also removed events with $1.17 \leq \mathrm{M}\left(\mathrm{p} \pi^{+}\right) \leq 1.29$. The resulting $\mathrm{n}^{+}{ }^{+}$and $\mathrm{pn}^{\mathrm{o}}$ effective mass distributions are shown in Figs. 1(a) and 2(a). Figures 1 (b) and $2(\mathrm{~b})$ represent events with the further selection $\cos \theta \geq 0.975$, where $\theta$ is the production angle, in the total center-of-mass, of the $\mathrm{n} \pi^{+}$or $\mathrm{p} \pi^{\circ}$ combined mass. In the $n \pi^{+}$. system, there appears to be an enhancement at 1.4 $\mathrm{BeV} / \mathrm{c}^{2}$ in addition to $\mathrm{N}^{*^{++}}(1238), \mathrm{N}^{*}(1510)$, and $\mathrm{N}^{+}(1688)$ peaks. The peak at $1.4 \mathrm{BeV} / \mathrm{c}^{2}$ and $\mathrm{N}^{+}(1238)$ are most conspicuous at small production angles while $\mathrm{N}^{*}(1510)$ and $\mathrm{N}^{+}(1688)$ exhibit a lesser degree of peripheralism. It is this peripheral property that enables the $1.4 \mathrm{BeV} / \mathrm{c}^{2}$ peak to be resolved from $\mathrm{N}^{*}(1510)$. The appearance of a similar effect at $1.4 \mathrm{BeV} / \mathrm{c}^{2}$ in the $\mathrm{p} \pi^{0}$ mass distribution is much less clear. However, isospin conservation requires that the rate of decay into $\mathrm{p} \pi^{\circ}$ of an $I=1 / 2$ resonance be half of the rate of decay into $\mathrm{n} \pi^{+}$. Hence, we place more significance on the results obtained from the $\mathrm{n} \pi^{+}$ system.

A one-pion exchange (OPE) calculation of the $n \pi^{+}$mass spectrum was made; it included contributions from $n \pi^{+}$production at one vertex and reflection from the other diagram, viz., $\mathrm{p} \pi^{+}$production at a vertex. We find that interference 
between these two processes amounts to about $4 \%$ of the total cross section, and only negligibly alters the shapes of the mass distributions. The four-momentum transfer dependence was taken to be an exponential $\mathrm{e}^{\alpha(\mathrm{m}) \mathrm{t}}$ with the function $\alpha(\mathrm{m})$ approximated by constants in the various mass intervals. These were determined by fitting the experimental $t$ distributions. This parametrization, when limited to small $t$, gives an adequate representation of the $t$-dependence that takes into account variations with $\pi$-nucleon mass as induced, for example, by the Dürr and Pilkuhn type of vertex correction. 5

The solid line drawn onto Fig. 1(b) gives the result of an OPE calculation which incorporates the experimental restrictions. Except for a 2.0 standard deviation peak near $1.4 \mathrm{BeV} / \mathrm{c}^{2}$, it gives a good fit to the data, in particular accounting for the production of $\mathrm{N}^{+}(1238), \mathrm{N}^{*}(1510)$, and $\mathrm{N}^{+}(1688)$. The events remaining above the OPE curve, when fitted to a Breit-Wigner resonance shape, yield a mass and width $\mathrm{M}=1.40 \pm 0.06 \mathrm{BeV} / \mathrm{c}^{2}$ and $\Gamma=0.11 \pm 0.06 \mathrm{BeV}$. The cross section is estimated to be $0.14 \pm 0.07 \mathrm{mb}$. Of course the OPE result includes the effect of $\mathrm{N}^{*}(1400)$ production via pion exchange, since the $\mathrm{P}_{11}$ resonance is included naturally. The calculation shows that the OPE contribution to $\mathrm{N}^{*}(1400)$ production does not produce a peak in the $n \pi^{+}$mass spectrum. In order to make a pessimistic estimate of $\mathrm{N}^{*}(1400)$ production we have also, within the associated errors, varied the coefficients $\alpha(m)$ so that the contribution of the $\mathrm{p} \pi^{+}$production diagram to the $\mathrm{n}^{+}$mass spectrum near $1.4 \mathrm{BeV} / \mathrm{c}^{2}$ is maximized. Therefore, the cross section for $\mathrm{N}^{*}(1400)$ production estimated above is expected to be smaller than the actual value.

To estimate the total resonance production cross sections, we have fitted the $n \pi^{+}$mass distribution of Fig. $1(b)$ to the form

$$
\begin{gathered}
F(m)=\left[a+\sum_{i} b_{i} \times(B W)_{i}\right] \times \phi(m)+R(m) \\
-4-
\end{gathered}
$$


where $(\mathrm{BW})_{\mathbf{i}}$ represents the Breit-Wigner expression for the $i^{\text {th }}$ resonance, $\phi(\mathrm{m})$ is the peripheral phase space for $n \pi^{+}$production at one vertex, ${ }^{6} \mathrm{R}(\mathrm{m})$ is the reflection from the corresponding diagram for $\mathrm{p} \pi^{+}$production at a vertex, and $\mathrm{a}, \mathrm{b}_{\mathrm{i}}$ are the intensity coefficients.

In fitting to the histogram of $1(\mathrm{~b})$, we allow the mass and the width of $\mathrm{N}^{*}(1400)$ to vary while constraining those of $\mathrm{N}^{*}(1238), \mathrm{N}^{*}(1510)$, and $\mathrm{N}^{*}(1688)$ to the known values. By this procedure, we find the mass, the width, and the production cross section of $\mathrm{N}^{*}(1400)$ to be $\mathrm{m}=1.39 \pm 0.02 \mathrm{BeV} / \mathrm{c}^{2}, \Gamma=0.15 \pm 0.06 \mathrm{BeV}$, and $\sigma\left[\mathrm{N}^{*}(1400) \rightarrow \mathrm{n} \pi^{+}\right]=0.27 \pm 0.13 \mathrm{mb}$. The mass and width agree with those determined from the missing mass spectra and are consistent with the phase shift analysis results.

A similar procedure was applied to the $\mathrm{p} \pi^{0}$ mass spectrum. The fit is represented by the solid curve in Fig. 2(b). We estimate that $\sigma\left[N^{*}(1400) \rightarrow p \pi^{0}\right]=$ $0.11 \pm 0.09$.

The cross sections for production of $\mathrm{N}^{+}(1238), \mathrm{N}^{+}(1510)$, and $\mathrm{N}^{+}(1688)$, as presented in Table I, are consistent with the values obtained from the missing mass experiments. ${ }^{7}$ Furthermore, the branching ratio between the $\mathrm{n} \pi^{+}$and $\mathrm{p} \pi^{\circ}$ channels for each of these resonances is consistent with that expected from the conservation of isospin. This provides a measure of the reliability of the results obtained by the procedures employed.

There is no evidence of $\mathrm{N}^{*}(1400)$ in the $\left(\mathrm{p} \pi^{+}\right)$system where an $\mathrm{I}=3 / 2$ effect would be largest. Also, the branching ratio $\sigma\left[\mathrm{N}^{*}(1400) \rightarrow \mathrm{n} \pi^{+}\right] / \sigma\left[\mathrm{N}^{*}(1400) \rightarrow \mathrm{p} \pi^{\mathrm{O}}\right]$ is consistent with value 2.0 . The $N^{*}(1400)$ can hence be assigned isospin $1 / 2$.

We find $\sigma\left[\mathrm{N}^{*}(1400) \rightarrow \mathrm{n} \pi^{+}+\mathrm{p} \pi^{0}\right]=0.38 \pm 0.16 \mathrm{mb}$. With $\sigma\left[\mathrm{N}^{*}(1400) \rightarrow\right.$ all final states $]=0.60 \pm 0.14 \mathrm{mb}$ from the counter experiments, the elasticity is about 0.66 . Again, this is consistent with the conclusions of the phase shift 
analyses of elastic scattering. To complete the identification of the $N^{*}(1400)$ peak with the $P_{11}$ resonance, it is necessary to establish its spin and parity. Unfortunately, the large background to signal ratio in the $\mathrm{N}^{*}(1400)$ region precludes any investigation of the decay angular distribution, so that no spin-parity assignment of the partial waves causing the peak can be made.

Since the pion exchange production mechanism gives a good account of the production of resonances other than $\mathrm{N}^{*}(1400)$, some additional mechanism appears to be operative.

The $t$ distributions of all events under the $1.4 \mathrm{BeV} / \mathrm{c}^{2}$ peak fitted to the exponential form $\mathrm{e}^{\alpha \mathrm{t}}$ gives $\alpha=10.4 \pm 1.0 \mathrm{BeV}^{-2}$. The missing mass experiments ${ }^{3}$ indicate that when the non-resonance background events are properly subtracted, the value of $\alpha$ for $N^{*}(1400)$ alone is expected to be larger than $10.4 \mathrm{BeV}^{-2}$. This peripheral nature suggests the dominance of a one-particle or single Regge trajectory exchange mechanism. Therefore, if the excess of events in the $N^{*}(1400)$ above the OPE prediction corresponds to a resonance, then this resonance would be strongly coupled to a system other than $\pi$-nucleon. Similar conclusions follow from the energy dependence of $N^{*}(1400)$ production observed in missing-mass experiments. These show that the production cross section of $N^{*}(1400)$ remains almost constant with the incident proton momentum over the range of $6.0 \mathrm{BeV} / \mathrm{c}$ to $30.0 \mathrm{BeV} / \mathrm{c}$. This disagrees strongly with OPE model calculations which predict a rapid decrease in the cross section as the energy increases. A diffraction mechanism would provide a better description of such a process.

\section{ACKNOWLEDGMENTS}

We would like to thank Miss Mary Corey and Mr. William R. Gage for their programming efforts and also to express our appreciation to the scanning and measuring crews. 
FOO'TNOTES AND REFERENCES

1. L. D. Roper, Phys. Rev. Letters 12, 340 (1964);

A. Donnachie, R. G. Kirsopp and C. Lovelace, Phys. Letters $\underline{268,} 161$ (1968);

P. Bareyre, C. Bricman and G. Villet, Phys. Rev. 165, 1730 (1968).

The value of the mass is somewhat uncertain, and can be as low as about $1.4 \mathrm{BeV} / \mathrm{c}^{2}$.

2. The resonance is not seen directly in measurements of the $\pi^{ \pm}$total cross section as a function of incident momentum.

3. K. J. Foley et al., Phys. Rev. Letters 19, 397 (1967);

I. M. Blair et al., Phys. Rev. Letters 17, 789 (1966);

E. W. Anderson et al., Phys. Rev. Letters 16, 855 (1966);

C. M. Ankenbrandt et al., Nuovo Cimento 35, 1052 (1965);

G. Cocconi et al., Phys. Letters 8,134 (1964).

4. E. Gellert et al., Phys: Rev. Letters 17, 789 (1966).

5. H. P. Dürr and H. Pilkuhn, Nuovo Cimento 40A, 899 (1965).

6. The peripheral phase space for the production of $n \pi^{+}$at one vertex is obtained by putting an exponential factor $e^{\alpha t}$, where $t$ is the four-momentum transfer squared to the $\mathrm{n}^{+}$system, into the ordinary phase space integral. The quantities $\alpha=\alpha(\mathrm{m})$ are determined from the experimental $\mathrm{t}$ distributions for each mass interval.

7. If one of the parameters in the fit, namely the width of $N^{*}(1400)$, is fixed at the average value of $.15 \mathrm{BeV}$ quoted by the missing mass spectrometer experiments the uncertainty in the cross section for the $N^{*}(1400)$ production is halved, so that $\sigma\left[\mathrm{N}^{*}(1400) \rightarrow \mathrm{n} \pi^{+}\right]=.27 \pm .07 \mathrm{mb}$. The uncertainties in the cross sections for the production of other $\mathrm{N}^{*}$ resonances are reduced also by at least a factor of 1.5 . 


\section{TABLE CAPTION}

I. Resonance production cross sections in $\mathrm{mb}$.

\section{FIGURE CAPTIONS}

1. (a) Mass histogram for $\mathrm{n}^{+}$system with $\mathrm{N}^{*^{++}}$(1238) removed, proton going backward with respect to the beam, neutron going forward.

(b) Same as (a) but also with $\cos \theta\left(n \pi^{+}\right) \geq .975$. Solid line represents OPE prediction.

2. (a) Mass histogram for $\mathrm{p} \pi^{\mathrm{o}}$ system.

(b) Same as (a) but $\left|\cos \theta\left(\mathrm{p} \pi^{\circ}\right)\right| \geq .975$. Solid line represents fits to expression (4). 


\section{TABLE I}

Resonance Production Cross Sections in $\mathrm{mb}$

\begin{tabular}{|c|c|c|}
\hline Decay Mode & $\mathrm{n} \pi^{+}$ & $\mathrm{p} \pi^{\circ}$ \\
\hline $\mathrm{N}^{*}(1238)$ & $.28 \pm .08$ & $.52 \pm .13$ \\
$\mathrm{~N}^{*}(1400)$ & $.27 \pm .13$ & $.11 \pm .09$ \\
$\mathrm{~N}^{*}(1510)$ & $.15 \pm .09$ & $.08 \pm .05$ \\
$\mathrm{~N}^{*}(1688)$ & $.19 \pm .09$ & $.10 \pm .05$ \\
\hline
\end{tabular}




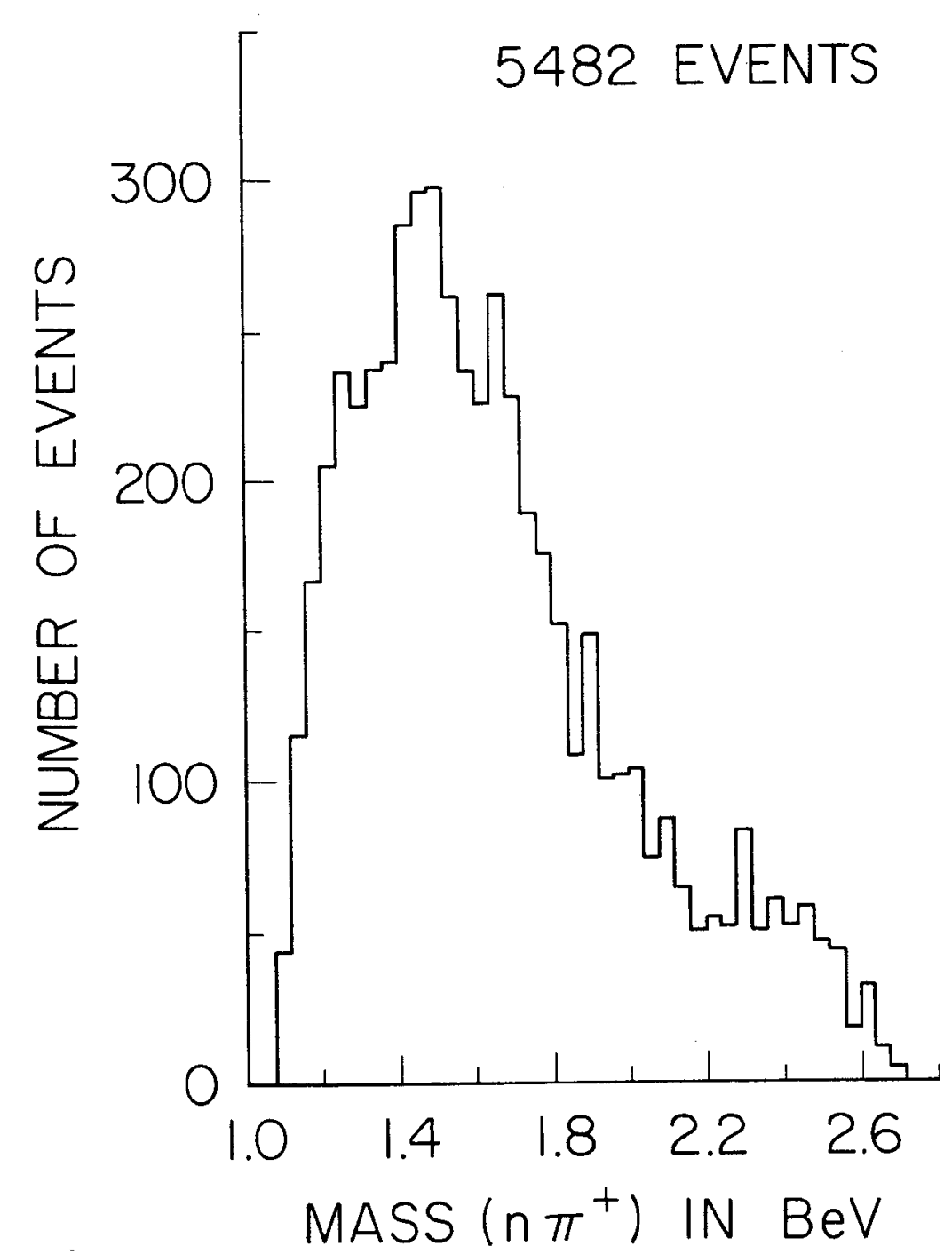

a.

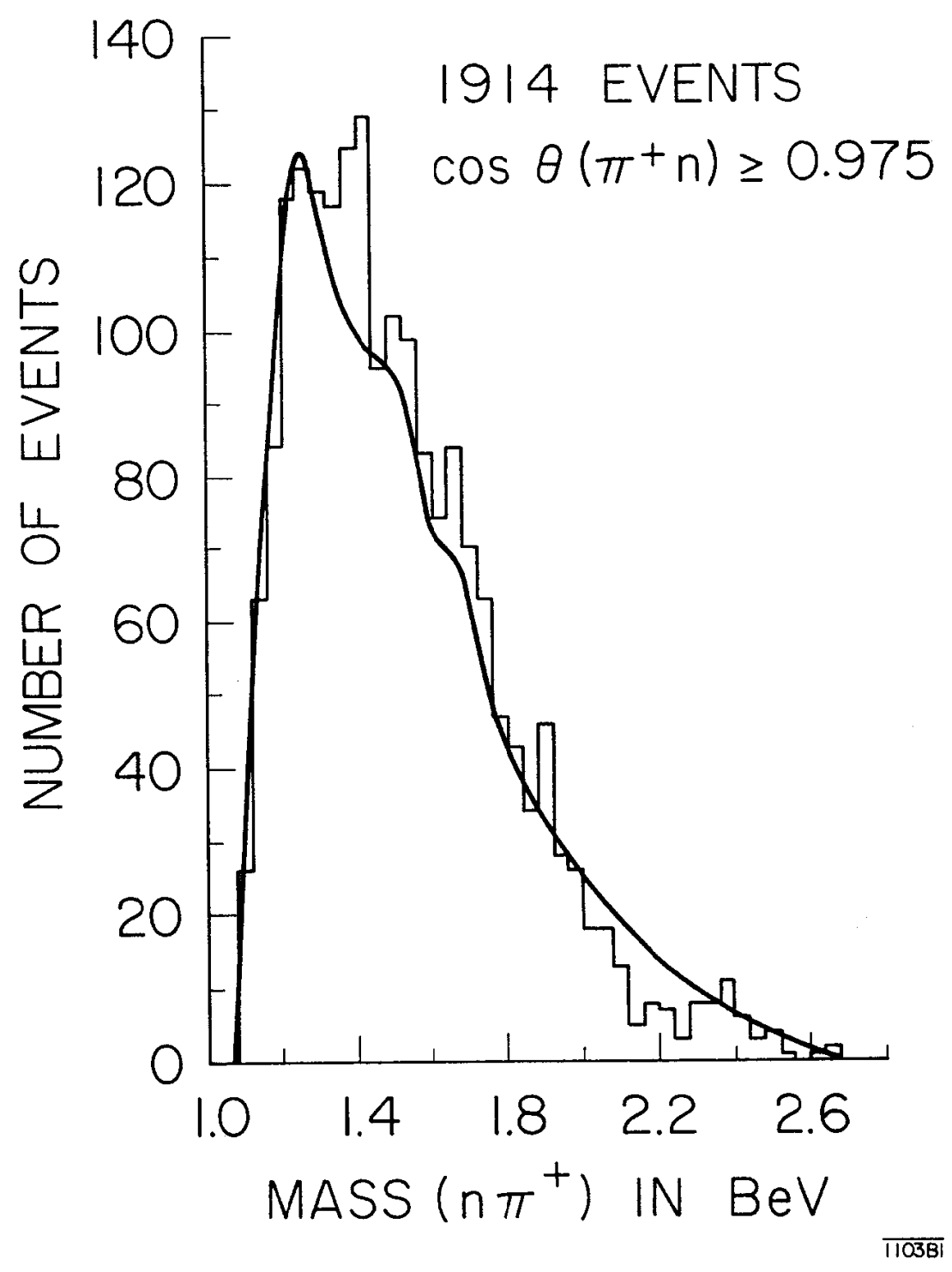

b.

Fig. 1 

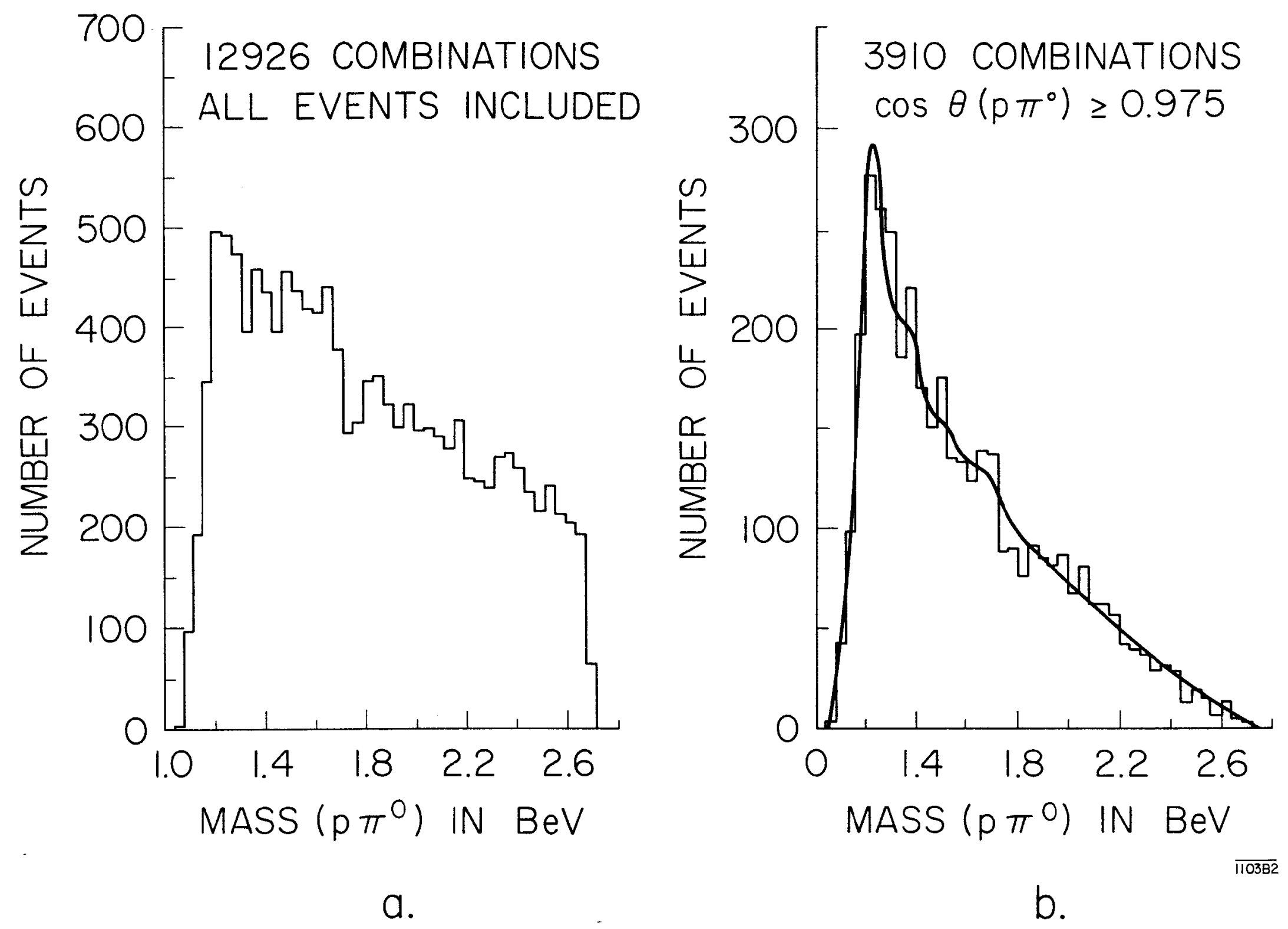

Fig. 2 